\title{
Symptoms, warning signs, and lifestyle choices associated with head and neck cancer
}

\section{Chin-Lung Kuo* Ming University, Taiwan, ROC \\ Challenges of working people in modern society}

Department of Otolaryngology, HsinChu Armed Forces General Hospital; Taoyuan Armed Forces General Hospital; Institute of Brain Science, National Yang-

Recently two of my friends developed cancer, and one of my patients was diagnosed with oral cancer. The devastating news shocked me and evoked sympathy for the plight of modern workers. They often face unreasonable expectations from supervisors, and can become overstressed from the desire to perform flawlessly at their job. Moreover, some are forced to work many overtime hours. This overwhelming pressure can lead to gastric and duodenal ulcers. After devoting decades of their lives to work, many of these individuals expect to have a comfortable retirement. Instead however, they encounter soaring prices and economic recession; therefore, as their savings diminish they are forced to keep working. If such an individual were to learn that they have developed cancer, they may not be able to handle the news.

\section{Head and neck cancer}

In Taiwan, cancer has been the leading cause of death for 33 consecutive years (since 1982). Furthermore, according to statistics compiled by the Ministry of Health and Welfare, the 2014 cancer mortality rate was more than twice as high as the mortality rate of heart disease, which was the second leading cause of death. Out of every 10 newly diagnosed cancer cases, 1-2 comprise head and neck cancer. Oral cancer was the fifth deadliest cancer in Taiwan in 2014 [1]. This is especially alarming given that, in 1994, when the incidence of oral cancer grew larger than that of nasopharyngeal cancer, it became the most common head and neck cancer, with an average of over 4,000 new cases appearing every year.

Head and neck cancer can originate in multiple locations. Types of head and neck cancers include oral cancer (primarily tongue and buccal cancers), oropharyngeal cancer, hypopharyngeal cancer, laryngeal cancer, salivary gland cancer, thyroid cancer, nasopharyngeal cancer, as well as nasal cavity and paranasal sinus cancer. Over $90 \%$ of head and neck cancers involve squamous cell carcinoma. These cancers are associated with genetics as well as hereditary, dietary, and environmental risk factors that are strongly linked to carcinogens, such as tobacco, alcohol, and betel nuts.

Indeed, over $90 \%$ of head and neck cancer patients habitually smoke, drink, or chew betel nuts. For example, in Taiwan, individuals who regularly consume all three of these substances are 123 times more likely to develop oral cancer than those who do not (Figure 1) [2]. Conversely, individuals who smoke and chew betel nuts are 89 times more likely to develop oral cancer; individuals who drink and chew betel nuts are 54 times more likely to develop oral cancer; individuals who drink and smoke are 22 times more likely to develop oral cancer; individuals who smoke are 18 times more likely to develop oral cancer; individuals who chew betel nuts are 28 times more likely to develop oral cancer, and individuals who drink are 10 times more likely to develop oral cancer. Clearly, betel nut chewing is the greatest risk factor of oral cancer [2].

\section{Oral cancer}

Different head and neck cancers have different clinical manifestations. Symptoms may appear individually or simultaneously, and generally begin with mucosal leukoplakias and/or erythroplakias. Erythroplakias in the mucosal surface are typically associated with a higher probability of subsequent cancer development than leukoplakias. Research showed that the 10-year cumulative malignant

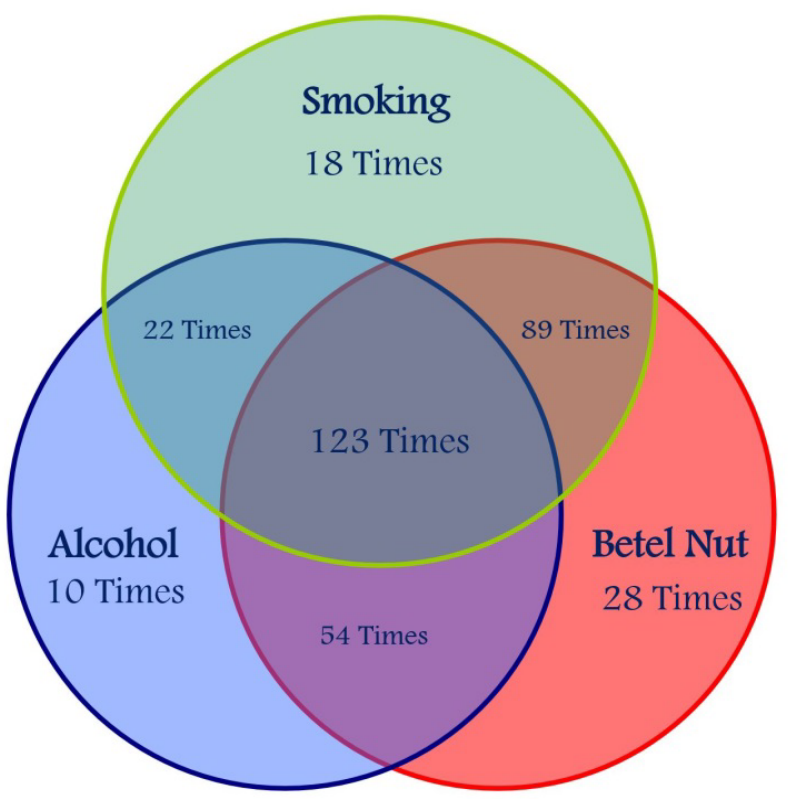

Figure 1. Venn diagram illustrating the risk of oral cancer associated with smoking, drinking, and betel nut chewing.

Correspondence to: Dr. Chin-Lung Kuo, Department of Otolaryngology, HsinChu Armed Forces General Hospital; Taoyuan Armed Forces General Hospital; Institute of Brain Science, National Yang-Ming University, Taiwan, Tel: 886-9-19681078, E-mail: drkuochinlung@gmail.com

Editor-in-Chief, Otorhinolaryngology Head and Neck Surgery.

Received: April 15, 2016; Accepted: May 06, 2016; Published: May 10, 2016 
transformation rates of leukoplakias range from $2.4 \%$ to $29 \%[3,4]$ however, for erythroplakias, the malignant transformation rates range between $14.3 \%$ and $66.7 \%[5,6]$. Oral cancer can also manifest as an ulcer that does not heal; therefore, if an ulcer or sore persists for more than two weeks, a biopsy should be performed to exclude the possibility of cancer.

\section{Oropharyngeal, hypopharyngeal, and laryngeal cancer}

The early symptoms of oropharyngeal cancer and hypopharyngeal cancer are not obvious or specific, thus early diagnosis is difficult regardless of how symptoms manifest. Most often, these symptoms involve a slight foreign body sensation in the throat; however, some patients present with a continuously and abnormally sore throat and/or earaches (referred pain). Additionally, in the early stages of cancer, the abundance of lymphoid tissue in the oropharynx and hypopharynx facilitates neck metastasis via the lymphatic system; therefore neck masses are another common early cancer symptom [7]. On the other hand, the indistinct early symptoms of oropharyngeal and hypopharyngeal cancers are easily confused with the symptoms of respiratory infections and gastroesophageal reflux disease (GERD). This presents a serious challenge and may lead to missed or delayed diagnosis, jeopardizing the prognosis.

In the advanced stage of cancer, patients may develop dysphagia and/or hoarseness. It is worth nothing that the author conducted a population-based study that used data from almost 40,000 individuals registered in the National Health Insurance Research Database of Taiwan. Findings revealed that patients who had previously been diagnosed with GERD were nearly four times more likely to subsequently suffer from oropharyngeal (3.58 times) or hypopharyngeal cancer (3.96 times) than those who had not previously received a GERD diagnosis [8]. Therefore patients with acid reflux should be referred to an otolaryngologist and undergo nasopharyngoscopies, in particular those who notice a foreign object sensation in the throat or who experience un-expellable phlegm, dysphagia, hoarseness upon waking, or unexplainable chronic coughing.

Conversely, laryngeal cancer presents hoarseness in early stages, hence early diagnosis is achievable. In later stages, laryngeal cancer is associated with dysphagia, dyspnea, and hemoptysis [9-11].

\section{Nasopharyngeal cancer}

The incidence rate of nasopharyngeal cancer in men in Taiwan is more than 10 times higher than that of men in the US and more than 20 times higher than that of men in Japan. Nasopharyngeal cancer is most prevalent in patients between the age of 40 and 50 and is more common in men than in women by a ratio of 3 to 1 [12]. The most common symptoms of nasopharyngeal cancer are neck masses. Indeed, at the time of diagnosis, approximately $80 \%$ of patients already have metastatic tumors in their neck. This is because the abundance of lymphoid tissue in the nasopharynx facilitates neck metastasis via the lymphatic system. Nose symptoms, including nasal obstruction, bloody nasal discharge, or blood-tinged sputum also indicate the possibility of nasopharyngeal cancer. Symptoms that occur in the ear, including tinnitus, aural fullness, and impaired hearing, may be warning signs that the nasopharyngeal cancer has invaded the Eustachian tube. Patients with unilateral aural symptoms should have their nasopharynx examined in order to exclude the possibility of nasopharyngeal cancer. An aggressive nasopharyngeal tumor may extend intracranially and cause neurological symptoms such as unilateral headaches, diplopia, facial numbness, or dysphagia.
Research teams at the Tri-Service General Hospital [13] and the Far Eastern Memorial Hospital [14] in Taiwan examined the correlation between allergic rhinitis and nasopharyngeal cancer using the National Health Insurance Research Database. Researchers from the TriService General Hospital found that patients with allergic rhinitis were 2.33 times more likely to develop nasopharyngeal cancer than those without allergic rhinitis. Conversely, researchers from the Far Eastern Memorial Hospital found that patients with nasopharyngeal cancer were 2.25 times more likely to have a history of allergic rhinitis than those without nasopharyngeal cancer. Moreover, the study conducted at the Far Eastern Memorial Hospital also found that patients with nasopharyngeal cancer were 3.83 times and 1.57 times more likely to have chronic and acute sinusitis in their medical history, respectively, than those without nasopharyngeal cancer [15]. All of these findings emphasize the importance of having an otolaryngologist perform regular nasopharynx examinations to check for tumors in patients who have suffered from allergic rhinitis and chronic or acute sinusitis.

\section{Cancer of unknown primary}

The neck contains 200 to 300 lymph nodes, and these serve as the first line of defense in the human immune system. Lymph nodes may swell in the event of inflammation or infection, such as that which occurs during colds, periodontal disease, or tonsillitis. Generally, middle-aged or elderly patients who present neck masses are first treated with antibiotics for 1 to 2 weeks. If the lumps do not subside in that time, then further investigation is required to rule out the possibility of malignancy.

According to the famous Merkulov's rule [16], also known as the rule of eighty, $80 \%$ of all non-thyroid neck masses are neoplasms; $80 \%$ of those neoplasms are malignant; and $80 \%$ of the malignant neoplasms are metastatic. Jones et al. also found that $80 \%$ of metastatic neoplasms originate from primary cancers of the head and neck [17]. Clearly, patients with masses in the neck should have an otolaryngologist perform a nasopharyngoscopy to eliminate the possibility of upper aerodigestive tract cancers with metastasis to the neck. When it is not possible to have a comprehensive nasopharyngoscopic examination performed by an otolaryngologist, a metastatic neck tumor can be erroneously excised in patients with nasopharyngeal cancer. The tumor excision may cause spreading of cancer cells in the neck and thereby influence patient prognosis. Furthermore, radiotherapy (with/ without chemotherapy), and not surgery, is the mainstay of treatment for patients with nasopharyngeal cancer. Therefore, neck mass excision before a comprehensive nasopharyngoscopic examination should be avoided.

Some patients' present large masses in the neck but no relevant clinical symptoms, yet biopsies confirm that these masses are metastatic tumors. Such cases are referred to as cancer of unknown primary (CUP) and account for roughly $3 \%$ to $9 \%$ of all cancers. CUP requires that computed tomography (CT), magnetic resonance imaging (MRI), or positron emission tomography (PET) scans be performed for the breasts, chest, abdomen, and pelvic cavity. Patients should also undergo upper gastrointestinal endoscopy and colonoscopy [18]. In general, most CUPs originate from the head and neck; however, cancers in the supraclavicular fossa can metastasize from thoracic malignancies, such as lung and esophageal cancers.

\section{Prevention of head and neck cancers}

Head and neck cancers most commonly affect middle-aged male patients, who are often the primary or sole economic provider in a 
family. Therefore, the development of head and neck cancer can force families to contend with financial instability and, as a result, they may not have the capacity to address the considerable medical expenses associated with cancer treatment. Although ongoing advances in medical technology have increased the opportunity for early diagnosis and treatment, public awareness about head and neck cancer remains very limited.

The best strategy for management of cancer is prevention rather than treatment. It is imperative that the public obtain a correct and thorough understanding of warning signs for head and neck cancers. Additionally, physicians need to keep the primacy of cancerrelated symptoms in mind when treating patients. A comprehensive investigation should be arranged in order to achieve early diagnosis and prompt treatment, thereby reducing the threat of head and neck cancer.

\section{References}

1. TMOHW (2015) Taiwan Ministry of Health and Welfare. Statistics on the causes of death.

2. Ko YC, Huang YL, Lee CH, Chen MJ, Lin LM, et al. (1995) Betel quid chewing, cigarette smoking and alcohol consumption related to oral cancer in Taiwan. J Oral Pathol Med24: 450-453. [Crossref]

3. Einhorn J, Wersall J (1967) Incidence of oral carcinoma in patients with leukoplakia of the oral mucosa. Cancer 20: 2189-2193. [Crossref]

4. Schepman KP, van der Meij EH, Smeele LE, van der Waal I (1998) Malignant transformation of oral leukoplakia: a follow-up study of a hospital-based population of 166 patients with oral leukoplakia from The Netherlands. Oral Oncol34: 270-275. [Crossref]

5. Lumerman H, Freedman P, Kerpel S (1995) Oral epithelial dysplasia and the development of invasive squamous cell carcinoma. Oral Surg Oral Med Oral Pathol Oral RadiolEndod79: 321-329. [Crossref]
6. Lapthanasupkul P, Poomsawat S, Punyasingh J (2007) Aclinicopathologic study of oral leukoplakia and erythroplakia in a Thai population. Quintessence Int38: e448-455. [Crossref]

7. Kuo CL, Lee TL, Chu PY (2013) Conservation surgery for hypopharyngeal cancer: changing paradigm from open to endoscopic. ActaOtolaryngol 133: 1096-1103. [Crossref]

8. Kuo CL, Chen YT, Shiao AS, Lien CF, Wang SJ (2015) Acid reflux and head and neck cancer risk: A nationwide registry over 13 years. AurisNasus Larynx 42: 401-405. [Crossref]

9. Wilson JA (2008) Does pharma fund the pipers? - Scottish Intercollegiate Guidelines Network (SIGN) guidelines 90 - diagnosis and management of head and neck cancer, October 2006. ClinOncol (R CollRadiol) 20: 661-663. [Crossref]

10. NCCN (2011) Clinical Practice Guidelines in Oncology, Head and Neck Cancers.

11. Vokes EE, Weichselbaum RR, Lippman SM, Hong WK (1993) Head and neck cancer. NEngl J Med328: 184-194. [Crossref]

12. Kuo CL, Ho DM, Ho CY (2014) Dural metastasis of nasopharyngeal carcinoma: rare, but worth considering. Singapore Med J55: e82-84. [Crossref]

13. Lin KT, Huang WY, Lin CC, Jen YM, Lin CS, et al. (2015) Subsequent risk of nasopharyngeal carcinoma among patients with allergic rhinitis: a nationwide population-based cohort study. Head Neck 37: 413-417. [Crossref]

14. Chung SD, Wu CS, Lin HC, Hung SH (2014) Association between allergic rhinitis and nasopharyngeal carcinoma: a population-based study. Laryngoscope 124:1744-1749. [Crossref]

15. Hung SH, Chen PY, Lin HC, Ting J, Chung SD (2014) Association of rhinosinusitis with nasopharyngeal carcinoma: a population-based study. Laryngoscope 124: 15151520. [Crossref]

16. Merkulov V, Petrov N (1997) The role of ultrasonography in the diagnosis of neck lymphadenopathies. Centr EastEurope J ORL-HNS2:28.

17. Jones AS, Cook JA, Phillips DE, Roland NR (1993) Squamous carcinoma presenting as an enlarged cervical lymph node. Cancer 72: 1756-1761. [Crossref]

18. Marur S, Forastiere AA (2008) Head and neck cancer: changing epidemiology, diagnosis, and treatment. Mayo ClinProc 83: 489-501. [Crossref]

Copyright: $(2016$ Kuo CL. This is an open-access article distributed under the terms of the Creative Commons Attribution License, which permits unrestricted use, distribution, and reproduction in any medium, provided the original author and source are credited. 